\title{
A Method of Speed Control during Over-ground Walking: Using a Digital Light-Emitting Diode Light Strip
}

\author{
Liang Huang ${ }^{1, a}$, Jie Zhuang ${ }^{2, b}$ and Yanxin Zhang ${ }^{1, c}$ \\ ${ }^{1}$ Department of Sport \& Exercise Science, the University of Auckland, New Zealand \\ ${ }^{2}$ School of kinesiology, Shanghai University of Sport, China \\ aliang.huang@auckland.ac.nz, bzhuangjiesh@163.com, 'yanxin.zhang@auckland.ac.nz
}

Keywords: Overground walking, LED, Gait analysis, Light strip, Microchip, PWM

\begin{abstract}
The purpose of this report was to introduce the design of a portable, inexpensive and programmable digital light-emitting diode (LED) system to control overground walking speed. The system includes a custom-made 10 meters digital LED strip and a digital microcontroller. By controlling the duration time of the power supply to each LED unit, a visible running lights signal can provide a visual cue for speed control. To evaluate this design, five subjects were asked to walk overground while following the LED visual cue at five different target speeds. The actual walking speeds were determined using Vicon motion capture system. The results of this evaluation showed a good match between the actual and desired speeds. The average percent difference was $2.51 \%$, measured over 250 walking trials by the subjects. $98 \%$ of trials had an percent difference smaller than $6.5 \%$, which is the maximum tolerated error within the literature. The inter-trial reliability for the LED speed control system ranged from 0.85 to 0.88 for faster speeds $(1.6 \mathrm{~m} / \mathrm{s}, 1.4 \mathrm{~m} / \mathrm{s})$, and slightly lower ranging from 0.74 to 0.79 at slower speeds $(1.2 \mathrm{~m} / \mathrm{s}, 1.0 \mathrm{~m} / \mathrm{s}, 0.8 \mathrm{~m} / \mathrm{s})$.
\end{abstract}

\section{Introduction}

Walking speed is a very important parameter in gait analysis, which affects the magnitude of various kinematic and kinetic measures during walking. There are various methods have been used for speed control. The most direct way is by using a motor driven treadmill for which the speed is adjustable. However, most treadmills are not equipped with embedded force plates, and ground reaction forces are not able to be collected. Although force-plate-integrated treadmills are commercially available, the cost of this type of treadmill is very high [1]. Moreover, it was reported that biomechanical differences exist between treadmill and overground walking [2].

For overground walking, the speed is usually controlled by several photocells or timing lights positioned along the walkway [3,4]. The average speed is calculated after each trial. The major disadvantage of these methods is that the participants' do not have a clear visual guide during walking, so more practice trials are needed to help them adjust their speed to match the target. Some other studies have asked subjects to walk at a wide range of speeds and only the trials that were closest ( $\approx 3 \%-6.5 \%$ errors) to the desired speed were chosen $[5,6]$. In a recent report, Espy [7] provided the subjects with a visible velocity target to follow during level walking. The target was a flag being driven at a constant velocity by a motor running parallel to walkway. The results showed that the velocity of walking was successfully manipulated. The disadvantages of this system are its low mobility and relative complexity.

There is a need to develop a portable, inexpensive speed control system. The purpose of this report was to introduce the design of a speed-adjustable and programmable digital light-emitting diode (LED) light system, which can be used to control subjects' speed during overground walking. The reliability and accuracy of this equipment was also analyzed by comparing desired speeds and actual walking speeds measured using motion capture system. 


\section{Methods}

$\boldsymbol{L E D s}$, a semiconductor light source, have been widely used in communication industry, media industry and interior lighting for different purposes. With the development of microchip technology, the visual effect of LED lights became diverse and programmable [8]. For example, LED lights can create the "running" effect, which means a LED light signal can be transmitted from one location to another along a strip smoothly. This motivated us to design a LED system to provide a visual cue for walking speed control.

Our LED light system includes a custom-made 10 meter digital LED strip and a digital microcontroller. The light strip is made up of $6.25 \mathrm{~cm} * 1.2 \mathrm{~cm} * 0.35 \mathrm{~cm}$ thin circuit boards with two LED lights and a HL1606 microchip mounted on each board. The HL1606 microchip contains a four pin input (DI, CI, SI, LI), which receives and copies the datasheet from microcontroller (Fig. 1). The microchip signal can be transmitted through four output pins (DO, CO, SO, LO). In this way, two LED units are connected and subsequently the signal can be transformed between LED units.

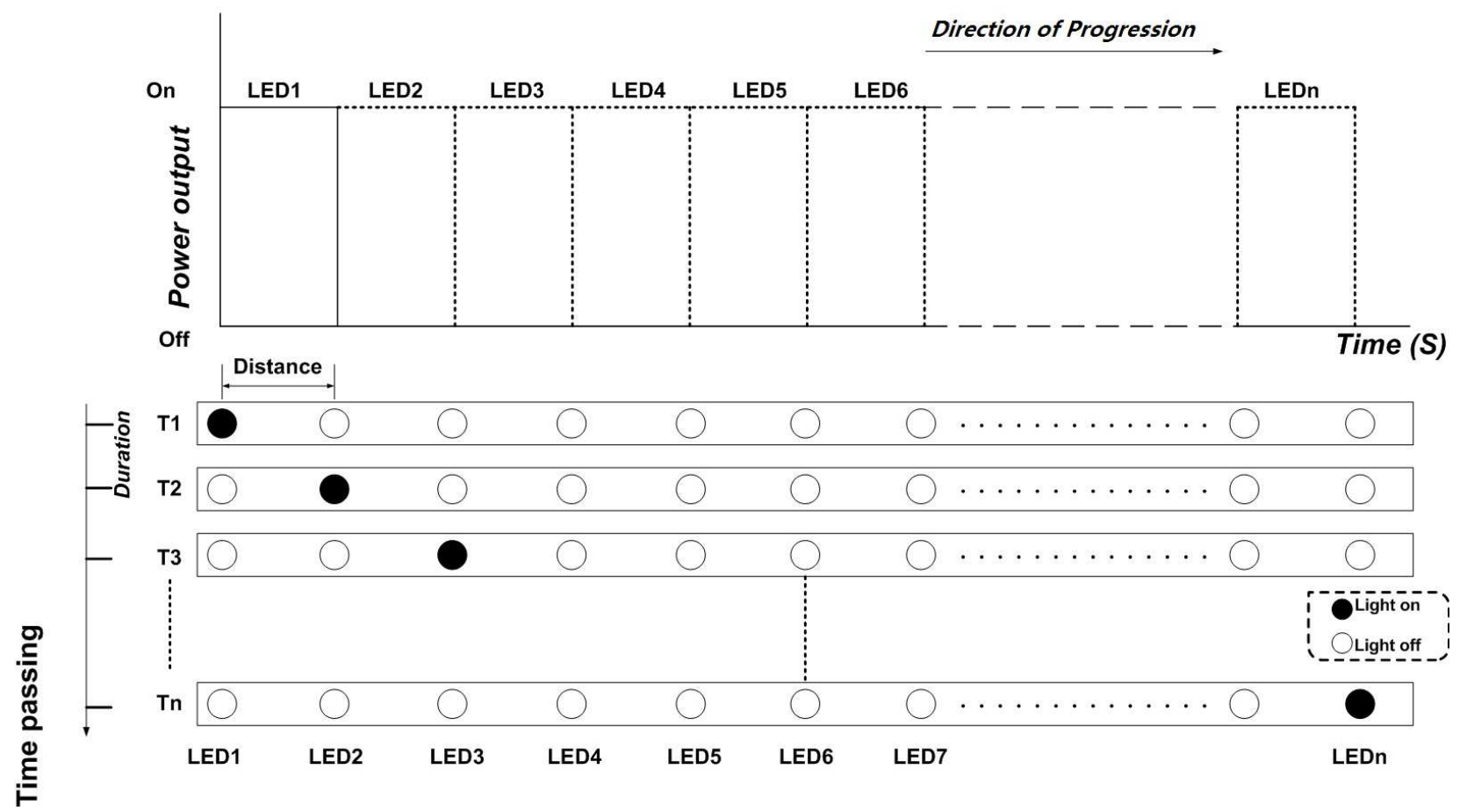

Fig. 1 Schematic of the "running" effect. The distance between each two adjacent LED unit is the same. The duration time of power supply for each LED is also the same.

$$
\text { Duration }=\mathrm{T}_{2}-\mathrm{T}_{1}=\mathrm{T}_{3}-\mathrm{T}_{2}=\ldots=\mathrm{T}_{\mathrm{n}}-\mathrm{T}_{\mathrm{n}-1} \text {. }
$$

A digital controller with STC90C52RC microcontroller (STC MCU Limited, China) was used to control the LED strip. By controlling LED power supply using Pulse Width Modulation (PWM) [9] technique, the first LED unit is turned on for a short duration of time then turned off at the same moment the next LED unit is turned on. By this way, the LED units are turning on quickly, one after another (Fig.2), creating a "running" effect by which the visual target (illuminated LED units) appears to move along the LED strip. 


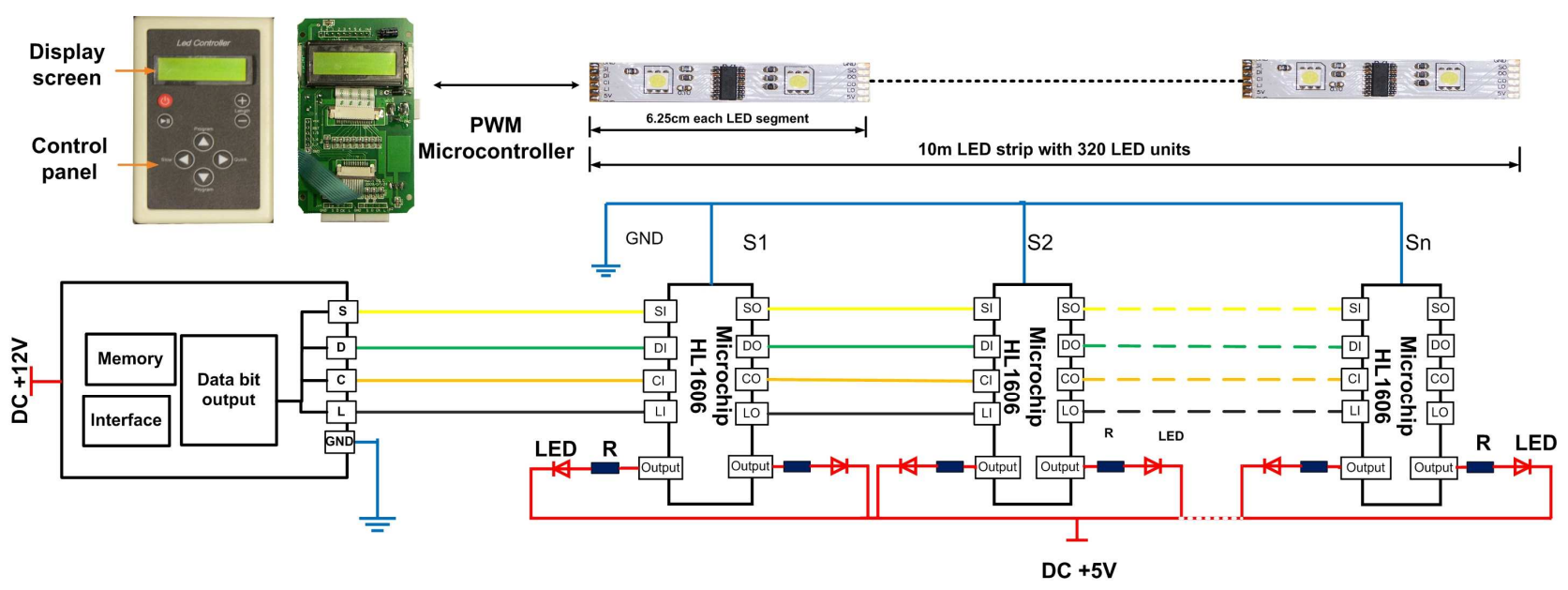

Fig. 2 Schematic of the LED system. The system includes a LED strip with HL1606 microchips and a PWM build-in microcontroller. The data is firstly sent and copied on the DI (data in), CI (clock in) and SI (speed clock input) lines. Under control of the LI (latch in), the data was pushed down the line to the next microchip through the DO (data out) pin, CO (clock out), SO (speed clock output) and LO

(latch out) pins. In this way, two LED units were connected and each one can be controlled individually.

To generate one target speed, one need the duration of the light on each LED unit and distance between adjacent LED units. By assigning a value to the duration in PWM datasheet, the speed can be calculated as the ratio of the distance between two LED units $(3.125 \mathrm{~cm})$ and the duration time. According to the source code of the PWM built-in module, the minimum duration of each LED unit is $0.008 \mathrm{~s}$. Each incremental increase in walking speed (total of 100 levels) this time duration increases by $0.001 \mathrm{~s}$. The equation is

Target Speed $=\frac{0.03125}{0.008+0.001 \times\left(N_{\text {level }}-1\right)} \mathrm{m} / \mathrm{s}$

The LED speed ranges from $0.10 \mathrm{~m} / \mathrm{s}$ to $3.90 \mathrm{~m} / \mathrm{s}$, which can cover the possible human walking speed range. In addition, the PWM module enables the user to set the number of LED units working as a group, that is, we can select either just one dot or a beam of light moving forward.

An Evaluation of method was designed to evaluate the accuracy of controlling walking speed control using the LED system. Experiments were carried out at the Biomechanics Laboratory of the University of Auckland. The experiment protocol was approved by The University of Auckland's Human Research Ethics committee. Five healthy subjects, (age: 24.8 \pm 3.6 years, height: $166.2 \pm 7.5$ $\mathrm{cm}$, three males and two females) were each asked to walk down a 10 meter walkway at five given speeds $(0.8 \mathrm{~m} / \mathrm{s}, 1.0 \mathrm{~m} / \mathrm{s}, 1.2 \mathrm{~m} / \mathrm{s}, 1.4 \mathrm{~m} / \mathrm{s}$ and $1.6 \mathrm{~m} / \mathrm{s})$ by following the light cue. The 10 meters digital LED light strip was installed on the side of the walkway and connected to the digital controller and power supply (12 volt Direct-Current). In order to increase the visual target, we set 16 serial LED units working together. The reflective markers were placed on the heel and the toe positions of the shoes (Fig. 3). The eight cameras Vicon motion analysis system (Oxford Metrics, UK) was used to record the displacement of the markers in three-dimension at a frequency of $100 \mathrm{~Hz}$. Ground reaction forces were measured by two in-ground AMTI force plates at a frequency of $1000 \mathrm{~Hz}$. Heel-strike and toe-off times were detected from the force plate data with a threshold value of $20 \mathrm{~N}$.

During each trail, the visual target (the set of 16 LED units), moved along the LED strip at the desired walking speed. The subjects were able to view the lights running in front of them without bending their heads (Fig. 3). Before capture, the participants carried out 5 to 10 times of practices at each LED-controlled speed. A researcher was walking along side to help subjects to match the speed during practices. After participants become familiar with the speed, they then performed ten 
successful walking at each desired speed by themselves. The stride length was determined by measuring the distance between heel marker positions during consecutive ground contacts of the same foot. The stride time was determined by measure the time from heel strike to heel strike of same foot. The timing of each heel strike was obtained from the forceplate data. The actual walking speed was computed as stride time divided by stride length. All data was processed by using Vicon Workstation software (Oxford Metrics, UK).

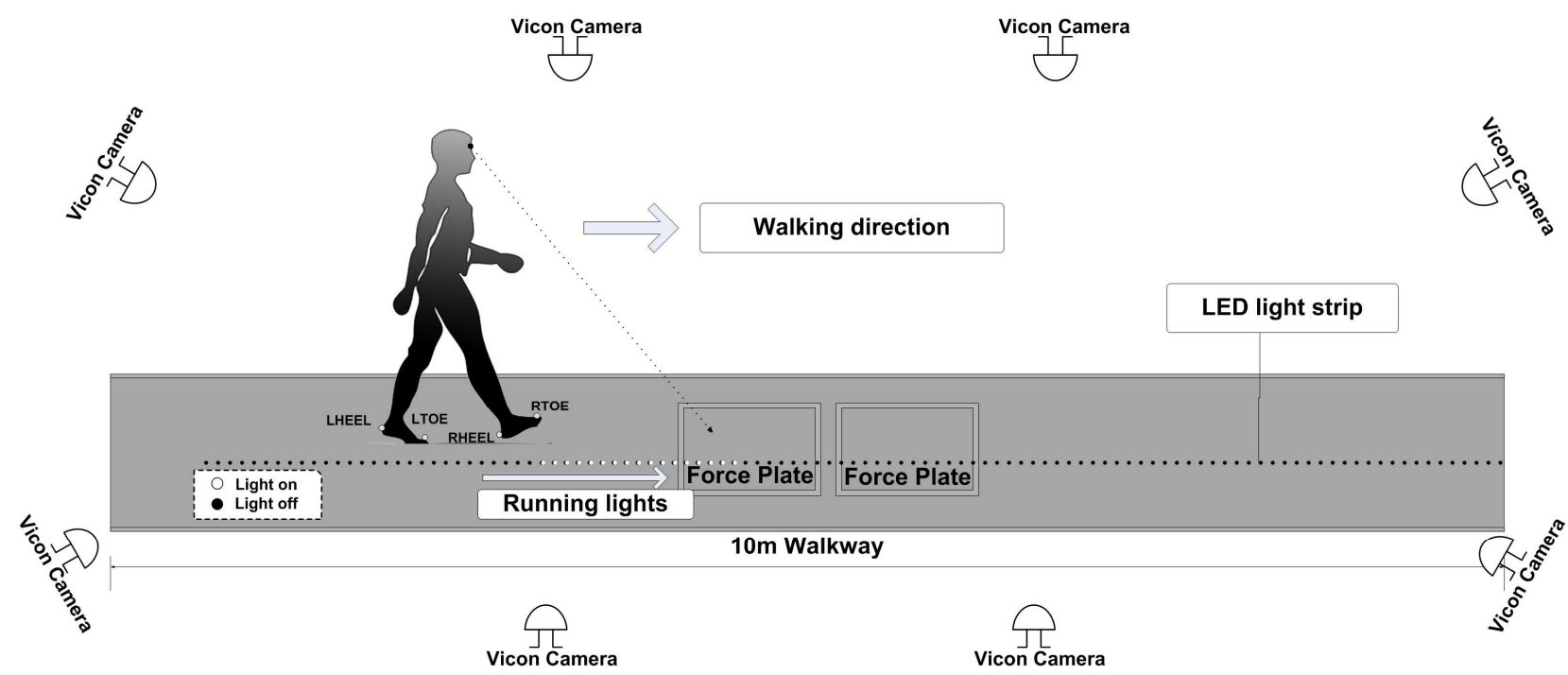

Fig. 3 The experimental set-up of the evaluation tests. The subjects followed the running lights along the $10 \mathrm{~m}$ walkway with two force-plates. Toe and heel markers were positioned to determine the feet segment.

The percent difference was calculated to explain the validity of each walking speed using the following equation:

Difference $\%=\frac{\left|V_{\text {measured }}-V_{\text {target }}\right|}{V_{\text {target }}} \times 100 \%$

Where $\mathrm{V}_{\text {measured }}$ is the measured walking speed using Vicon system and $\mathrm{V}_{\text {target }}$ is the LED target speed. A one way ANOVA was used to determine whether difference $\%$ was significant different between each two speeds. Intra-class correlation coefficient (ICC) was also calculated to test the reliability of the LED speed control system, by using SPSS software. A p-value of $<0.05$ was considered to be statistically significant.

\section{Results}

The average measured speed and percent difference values for each subject are shown in Table 1 . The measured percent difference ranged from $0.01 \%$ to $7.55 \%$. A statistically significant difference in measured percent difference was only seen between the $0.8 \mathrm{~m} / \mathrm{s}$ group and $1.2 \mathrm{~m} / \mathrm{s}$ group $(\mathrm{p}=0.011)$. There were no statistical differences found in the other 9 pairs of data. Over the 250 trials of the five subjects, $64.4 \%(161 / 250)$ of the trials had the percent difference of less than $3 \%, 33.6 \%(84 / 250)$ between $3 \%$ and $6.5 \%$, and only $2 \%(5 / 250)$ had the percent difference larger than $6.5 \%$. 
Table 1 The measured speed and percent difference for five subjects (Subject 1-5) at five different speeds (means $\pm \mathrm{SD}$ ), performing 10 walks for each speed.

\begin{tabular}{cccccc}
\hline & \multicolumn{5}{c}{ Difference $\%$} \\
\cline { 2 - 6 } & $0.8 \mathrm{~m} / \mathrm{s}$ & $1.0 \mathrm{~m} / \mathrm{s}$ & $1.2 \mathrm{~m} / \mathrm{s}$ & $1.4 \mathrm{~m} / \mathrm{s}$ & $1.6 \mathrm{~m} / \mathrm{s}$ \\
\hline Subject1 & $2.14 \pm 1.60$ & $2.03 \pm 1.42$ & $2.56 \pm 1.84$ & $1.93 \pm 1.52$ & $2.56 \pm 1.63$ \\
Subject2 & $2.38 \pm 1.77$ & $3.51 \pm 2.45$ & $3.69 \pm 1.80$ & $4.24 \pm 2.22$ & $1.28 \pm 1.32$ \\
Subject3 & $1.29 \pm 1.38$ & $1.13 \pm 1.15$ & $2.68 \pm 1.30$ & $2.07 \pm 1.07$ & $2.86 \pm 1.64$ \\
Subject4 & $3.14 \pm 2.05$ & $4.58 \pm 1.87$ & $3.36 \pm 1.54$ & $1.83 \pm 1.08$ & $2.76 \pm 1.63$ \\
Subject5 & $2.79 \pm 1.46$ & $1.82 \pm 0.69$ & $1.32 \pm 1.03$ & $1.99 \pm 1.83$ & $2.26 \pm 1.79$ \\
overall & $2.29 \pm 1.74$ & $2.67 \pm 2.06$ & $2.84 \pm 1.65$ & $2.37 \pm 1.72$ & $2.40 \pm 1.62$ \\
\hline
\end{tabular}

The ICC for the LED speed control system at five speeds, ranging from 0.85 to 0.88 for fast speeds level $(1.6 \mathrm{~m} / \mathrm{s}, 1.4 \mathrm{~m} / \mathrm{s})$, and slightly lower ranging from 0.74 to 0.79 at slower speeds $(1.2 \mathrm{~m} / \mathrm{s}, 1.0$ $\mathrm{m} / \mathrm{s}, 0.8 \mathrm{~m} / \mathrm{s})$.

\section{Discussion}

The LED system appears to be effective for speed control. All the subjects were able to follow the visible running lights signal, resulting in a good match of each desired speed. The average percent difference of this LED speed control system was $2.58 \%$. Based on the literature, two criteria have been proposed to assess the validity of walking speed based on error, the first being less that 3\% [6], and the other less than $6.5 \%$ error is considered valid [5]. Under these criteria, $63 \%$ and $97.5 \%$ of trials, respectively, are valid. The repeatability results for preferred walking speed were in the excellent range.

The result shows that compared to existing speed control system, the LED system has similar performance in terms of reliability and validity. Compared with the motor driven system [7], the LED system is easier to install or move. Thus walking speeds can be controlled directly without additional calculations, human resources and time are saved.

In studies which investigate both mechanical and metabolic cost of walking, these two parts are usually tested separately if force-plate-integrated treadmills are not available. In this case, the flexibility of the LED light strip enables itself to be wired to form a digital track that has built in force-plates. The subjects' walking speed can be kept steady under the guiding of the lights. Then the kinematic, kinetic and metabolic data can be measured simultaneously.

Although it is expected that the LED speed control system will improve present experimental methodologies of gait analysis, evidence is also needed to evaluate whether other population groups are able to follow the lights to achieve target velocities. In present study design, subjects were allowed to slightly look down at the running light during walking, in order to keep the pace. This is a potential issue that could affect the gait. It suggested that the LED strip can be raised to the subject's eye level in future experimental set-up. Because of its flexibility and portability, the LED strip system is easy to be elevated and supported by several rock stands, and the height is also adjustable. Another factor worth further consideration is that providing a visual cue could affect the gait of many pathologies including Parkinson's disease and therefore give a false impression of their gait. During walking in healthy subjects without any cognition problem, however, it is not expected to be a major concern.

\section{Acknowledgement}

This research was supported by Key Laboratory of Exercise and Health Sciences of Ministry of Education, Shanghai University of Sport. This work was also supported by Program for New Century Excellent Talents in University (NCET). 


\section{References}

[1] J. Leitch, et al., "Identifying gait events without a force plate during running: a comparison of methods," Gait Posture, vol. 33, pp. 130-2, Jan 2011.

[2] S. J. Lee and J. Hidler, "Biomechanics of overground vs. treadmill walking in healthy individuals," J Appl Physiol, vol. 104, pp. 747-55, Mar 2008.

[3] S. Vallabhajosula, et al., "Do lower-extremity joint dynamics change when stair negotiation is initiated with a self-selected comfortable gait speed ?" Gait \& Posture, 2011.

[4] P. Van de Walle, et al., "Age-related changes in mechanical and metabolic energy during typical gait," Gait Posture, vol. 31, pp. 495-501, Apr 2010.

[5] B. C. Bennett, et al., "Angular momentum of walking at different speeds," Hum Mov Sci, vol. 29, pp. 114-24, Feb 2010.

[6] M. Q. Liu, et al., "Muscle contributions to support and progression over a range of walking speeds," Journal of biomechanics, vol. 41, pp. 3243-3252, 2008.

[7] D. D. Espy, et al., "Control of center of mass motion state through cuing and decoupling of spontaneous gait parameters in level walking," J Biomech, vol. 43, pp. 2548-53, Sep 172010.

[8] R. D. Dupuis and M. R. Krames, "History, development, and applications of high-brightness visible light-emitting diodes," Journal of Lightwave Technology, vol. 26, pp. 1154-1171, 2008.

[9] A. M. Trzynadlowski, Introduction to modern power electronics: Wiley, 2010.

[1] J. van der Geer, J.A.J. Hanraads, R.A. Lupton, The art of writing a scientific article, J. Sci. Commun. 163 (2000) 51-59. 